\title{
Sexting: percepções de adolescentes sobre 0 fenômeno e acerca do papel das relações familiares
}

\section{Sexting: adolescents' perceptions on the phenomenon and about the role of family relationships}

\section{Sexting: percepciones de adolescentes sobre el fenómeno y acerca del papel de las relaciones familiares}

\author{
André Tavares Cardoso* \\ Universidade do Vale do Rio dos Sinos - Unisinos, São Leopoldo, Rio Grande do Sul, \\ Brasil
}

\section{Denise Falcke**}

Universidade do Vale do Rio dos Sinos - Unisinos, São Leopoldo, Rio Grande do Sul, Brasil

\section{Clarisse Pereira Mosmann***}

Universidade do Vale do Rio dos Sinos - Unisinos, São Leopoldo, Rio Grande do Sul, Brasil

\begin{abstract}
RESUMO
Pesquisas sobre sexting vêm abordando quantitativamente aspectos da individualidade dos adolescentes, desconsiderando suas percepções. Portanto, o objetivo dessa pesquisa qualitativa e descritiva foi conhecer a percepção de adolescentes sobre o sexting e sobre o papel das relações familiares no fenômeno. Foram realizadas entrevistas semiestruturadas com seis adolescentes entre 14 e 17 anos de uma escola da região metropolitana de Porto Alegre, com ou sem envolvimento em sexting. A partir da análise de conteúdo, os resultados mostraram que o fenômeno foi descrito como comum e não negativo. Entretanto, os participantes o descreveram como problemático caso ocorra o compartilhamento não autorizado. 0 exemplo dos pais e o diálogo na família foram percebidos como influenciadores de sexting ativo. Sugere-se que educadores, pais e terapeutas não o qualifiquem pejorativamente. Também que ampliem a comunicação sobre sexualidade para além do caráter informativo, bem como a discussão dos riscos de exposição que o compartilhamento não autorizado do sexting acarreta.

Palavras-chave: sexting, adolescência, família.
\end{abstract}

\section{ABSTRACT}

Sexting research has quantitatively addressed aspects of adolescent individuality, disregarding their perceptions. Therefore, the purpose of this qualitative and descriptive research was to know the adolescents' perception about sexting and the role of family relationships in the phenomenon. Semi- 
structured interviews were conducted with six adolescents between 14 and 17 years of age from a school in the metropolitan region of Porto Alegre city, with or without involvement in sexting. From the content analysis, the results showed that the phenomenon was described as common and nonnegative. However, participants described it as problematic if unauthorized sharing occurs. Parents' example and the dialogue in the family were perceived as influencers of active sexting. It is suggested that educators, parents and therapists do not qualify the phenomenon pejoratively. Also, they should extend communication about sexuality beyond the informational aspect, as well as the discussion of the exposure risks that the unauthorized sharing of sexting entails.

Keywords: sexting, adolescence, family.

\section{RESUMEN}

Las encuestas sobre sexting vienen abordando cuantitativamente aspectos de la individualidad de los adolescentes, desconsiderando sus percepciones. Por lo tanto, el objetivo de esta investigación cualitativa y descriptiva fue conocer la percepción de adolescentes sobre el sexting y del papel de las relaciones familiares en el fenómeno. Se realizaron entrevistas semiestructuradas con seis adolescentes entre 14 y 17 años de una escuela de la región metropolitana de Porto Alegre, con o sin participación en sexting. A partir del análisis de contenido, los resultados mostraron que el fenómeno fue descrito como común y no negativo. Sin embargo, los participantes lo describieron como problemático si se produce un intercambio no autorizado. El ejemplo de los padres y el diálogo en la familia fueron percibidos como influyentes de sexting activo. Se sugiere que los educadores, padres y terapeutas no lo califiquen peyorativamente. También que amplíen la comunicación sobre sexualidad más allá del carácter informativo, así como la discusión de los riesgos de exposición que el compartir no autorizado del sexting acarreta.

Palabras clave: sexting, adolescência, família.

O advento das novas tecnologias de comunicação por meio da internet trouxe mudanças na forma de interação social. Além do contato face a face, as pessoas passaram a se relacionar também de forma virtual, especialmente os jovens e adolescentes (Assunção \& Matos, 2014), que são os maiores usuários da internet via celulares e smartphones, tanto no Brasil, conforme o Instituto Brasileiro de Geografia e Estatística [IBGE] (2018), quanto em outros países (Korenis \& Billick, 2014; Strassberg, McKinnon, Sustaíta, \& Rullo, 2013).

Dentre as diferentes formas de uso do smartphone, está a troca de conteúdo sexual por meio de textos, imagens ou vídeos. Quando alguém produz e envia ou recebe conteúdos sexuais seus ou de outra pessoa, a literatura define esse comportamento como sexting (Rice et al., 2012; Rood, Thackeray, Letson, Leder \& Berlan, 2015). Tal comportamento tem crescido e se tornado comum em diferentes tipos de relacionamentos interpessoais, incluindo namoro, encontros casuais e amizades, particularmente entre os adolescentes em idade 
escolar (Delevi \& Weisskirch, 2013; Drouin, Vogel, Surbey \& Stills, 2013; Strassberg et al., 2013).

Entretanto, as pesquisas internacionais são heterogêneas ao relacionarem aspectos como sexo, idade e os motivos que levam os jovens ao envolvimento em sexting. Alguns estudos descreveram que as meninas são quem mais produzem e enviam sexting (Burkett, 2015; Döring, 2014) e que os meninos são os que mais comumente recebem tal conteúdo (Dir, Coskunpinar, Steiner, \& Cyders, 2013; Gordon-Messer, Bauermeister, Grodzinski, \& Zimmerman, 2013). Temple e Choi (2014) diferenciaram tais comportamentos como sexting ativo e passivo. Definiram como sexting ativo 0 comportamento de produzir e enviar conteúdo sexual de si ou de outro, e como sexting passivo o comportamento de receber tal conteúdo ou ser assediado a produzi-lo.

Porém, de acordo com Englander (2012), a constatação de que as meninas são mais envolvidas em sexting ativo do que os meninos pode ser questionada, pois são elas que recebem mais pressão para produzir e enviar sexting. Além disso, Delevi e Weisskirch (2013) sugeriram que as mulheres são mais propensas do que os homens a se envolver em sexting dentro de um contexto de relacionamento íntimo e de confiança, com o fim de manter ou fortalecer o relacionamento. Já os homens, ao contrário das mulheres, parecem utilizar o sexting como meio para atrair alguém a uma possível atividade sexual futura.

Em relação à idade dos adolescentes, não há consenso na literatura se são os mais novos ou não os mais envolvidos em sexting. $\mathrm{Na}$ Inglaterra, os adolescentes mais velhos comparados aos mais novos estiveram mais envolvidos no fenômeno (Livingstone \& Görzig, 2014). O mesmo foi encontrado no estudo de Rice et al. (2012) nos EUA, mas em contrapartida, no mesmo país o sexting foi bastante prevalente entre os menores de 14 anos (Rood, Thackeray, Letson, Leder, \& Berlan, 2015). Sendo assim, cabe questionar se no contexto brasileiro isso se ocorre da mesma forma, uma vez que o uso do smartphone é comum entre adolescentes de diferentes faixas etárias. No presente estudo, a adolescência será compreendida entre as idades de 12 e 17 anos, conforme o que dispõe o Estatuto da Criança e do Adolescente - ECA, (Lei n. 8069, 1990).

Quanto aos motivos para o engajamento em sexting há aqueles ligados a um contexto de relacionamento e os que não estão. Entre os primeiros aparecem o esforço para ser sexy, como forma de diversão, flerte, ganhar atenção do parceiro como expectativa de iniciar atividade sexual, como resposta a um pedido da outra pessoa ou ao fato de ter recebido sexting da mesma (Drouin et al., 2013; Henderson \& Morgan, 2011).

Já os motivos que não envolvem namoro ou relacionamento afetivo aparecem como forma de brincadeira entre amigos, com objetivo de 
fortalecer a amizade, como uma moeda de popularidade (Albury \& Crawford, 2012; Ringrose, Livingstone, \& Harvey, 2012) e como forma de buscar novas sensações (Baumgartner, Sumter, Peter, Valkenburg, \& Livingstone, 2014). Também como uma necessidade de pertencimento grupal, busca por autonomia e sensação de onipotência (Macedo, Azevedo \& Castan, 2010; Steinberg \& Morris, 2001). Em uma pesquisa no México, adolescentes também citaram problemas de identidade sexual, baixa autoestima, perceber-se socialmente discriminado, ser aluno novo na turma e tentar impressionar a outro com uma tentativa de iniciar um relacionamento (Mejía-Soto, 2014).

Portanto, uma vez que o sexting pode servir como meio de interação tanto em um relacionamento amoroso quanto de amizade é importante pensar sobre a atitude do adolescente em relação ao fenômeno. Nos Estados Unidos, o estudo de Strassberg et al. (2013) com 606 adolescentes mostrou que os que viam o sexting como errado foram os que menos enviaram imagens de si mesmos em relação aos que o consideraram aceitável. Os participantes também descreveram como possíveis consequências do sexting ativo o recolhimento do celular, suspensão ou expulsão escolar, penas para pornografia, prisão, prestação de serviços comunitários e multa.

Cabe notar que a percepção dos adolescentes a respeito de tais consequências recaiu sobre a possibilidade de serem pegos e não de estarem envolvidos em sexting, revelando que a prática do sexting em si não foi vista como inadequada, mas sim a divulgação ou o compartilhamento não autorizado das mensagens. Isso se coaduna com o que descreveram adolescentes portugueses, de que a exposição da vida publicamente era o que mais os preocupava no uso intenso da internet (Assunção \& Matos, 2014).

Entretanto, algumas pesquisas também demonstraram que o envolvimento em sexting pelos adolescentes esteve associado a problemas emocionais (Choiet al., 2016; Livingstone \& Görzig, 2014; Ouytsel, Ponnet, \& Walrave, 2017; Temple et al., 2014; Temple \& Choi, 2014), a comportamentos sexuais de risco, como o não uso do preservativo (Rice et al., 2012), uso de violência no namoro (Morelli, Bianchi, Baiocco, Pezzuti, \& Chirumbolo, 2016) e a implicações legais (Korenis \& Billick, 2014; Wolak, Finkelhor, \& Mitchell, 2012). Esses resultados estiveram relacionados tanto aos adolescentes que receberam quanto aos que enviaram tais imagens.

Em relação às questões emocionais, Temple et al. (2014) investigaram a associação entre sexting e saúde mental. Eles revelaram que o envolvimento em sexting esteve significativamente associado aos sintomas de depressão, impulsividade e uso de substâncias, mas não foi preditor de tais problemas. Pode ser que os adolescentes sob a influência de álcool e drogas fiquem mais desinibidos e vulneráveis ao envolvimento em sexting nessas 
circunstâncias. Talvez a relação entre sexting e o uso de substâncias seja espúria, possivelmente devido à existência de variáveis subjacentes compartilhadas entre os participantes, tais como amizades que favorecem envolvimento em atos infracionais ou características próprias da adolescência.

No que se refere aos comportamentos sexuais, Temple e Choi (2014) em seu estudo longitudinal descreveram que os adolescentes que enviaram imagens íntimas (sexting ativo) tiveram mais relações sexuais no ano seguinte comparados aos que não enviaram (sexting passivo). Isso leva a pensar que talvez a função do sexting ainda precise ser compreendida no que se refere ao desenvolvimento sexual dos adolescentes. De fato, a vivência da sexualidade na adolescência tem um papel normativo no desenvolvimento e por isso precisa ser estudada de forma integral e neutra, isto é, sem a pressuposição de que ela traz apenas riscos (Tolman \& McClelland, 2011).

A partir disso, pode-se indagar qual o papel do sexting na adolescência, fase importante do ciclo vital que possui suas peculiaridades. Por exemplo, sabe-se que é um período de muitas transformações e descobertas e os adolescentes estão em pleno processo de mudança do corpo, que biologicamente deixa de ser um corpo infantil para tornar-se reprodutivamente apto (Bezerra, Queiroz, \& Oliveira, 2014). Eles também estão desenvolvendo novas formas de intimidade e são confrontados com a necessidade de aprender a regular o comportamento sexual. Assim, experimentam uma tensão entre o desejo de autossatisfação e o atendimento das expectativas sociais e familiares a respeito da sua sexualidade.

No Brasil, Oliveira, Béria e Schermann (2014) mostraram que os pais viam o início da vida sexual como símbolo de maturidade para os filhos, mas não para as filhas. Assim, visto que na família a sexualidade ainda é vista como tabu pelos pais (González, Orcasita, Carrillo, \& Palma-García, 2017; Sevilla, Sanabria, Orcasita, \& Palma, 2016), é importante conhecer o que o sexting representa aos adolescentes.

Muitos pais não conversam com os filhos sobre o tema da sexualidade por atribuírem um valor negativo a ele, pela dificuldade de aceitar a sexualidade dos filhos, por acreditarem que falar estimula os filhos a praticarem sexo (Gonçalves, Faleiro, \& Malafaia, 2013) e por se sentirem despreparados e tímidos para tratar do tema com eles (Sevilla et. al., 2016). Adiciona-se a isso o fato de que, na escola, muitas vezes a educação sexual se resume aos aspectos biológicos, reprodutivos e não supre as ansiedades dos adolescentes, desconsiderando as dimensões prazerosa e benéfica (Gonçalves et al., 2013). Especialmente no contexto atual brasileiro, em que a sexualidade e o sintagma ideologia de gênero (Junqueira, 2018) 
estão sendo discutidos (Projeto de lei da 'Escola sem Partido' que avança na Câmara, 2018).

Sevilla et al. (2016) descreveram em seu estudo que os pais tinham mais dificuldade em falar sobre sexo com seus filhos do que as mães. Quando aconteciam conversas sobre sexualidade, elas se concentravam na proteção das mulheres e na promoção da sexualidade para os homens e a maior fluência no diálogo se dava entre os subsistemas mãe-filha e pai-filho. Além disso, houve discrepância na percepção entre os pais e os adolescentes no sentido de que, para os pais, seria suficiente apenas mencionar de forma diretiva certos tópicos sobre sexualidade, enquanto a expectativa dos filhos era de que eles discutissem valores e dinâmicas de experiências sexuais. Isso talvez explique porque os amigos e a internet são as maiores fontes de informação sobre sexualidade entre os adolescentes (Gondim et al., 2015), o que aponta para a possibilidade de o uso das tecnologias ser mediador dessa troca de informações.

Visto que as famílias ainda têm dificuldades em abordar o tema sexualidade com seus filhos é importante investigar como, em relação ao sexting, a comunicação entre pais e filhos intermediaria o envolvimento destes no fenômeno. Sabe-se que a comunicação entre os subsistemas na família é fundamental para uma boa interação entre eles (Nichols \& Schwartz, 2007; Vasconcellos, 2013) e que o diálogo com os pais tem efeito protetor na vida dos adolescentes (Gomide et al., 2005; Tomé, Camacho, De Matos, \& Diniz, 2011). Por exemplo, Moscoso-Alvarez, Rodríguez-Figueroa, Reyes-Pulliza e Colon (2016) indicaram que a comunicação entre pais e filhos, teve um papel importante na saúde mental dos adolescentes de Porto Rico. Sendo assim, pode-se questionar se o mesmo ocorreria com o sexting. Talvez o fenômeno possa ser percebido por adolescentes como instrumento de comunicação familiar sobre sexualidade, assim como servir como fonte de informação sobre ela.

Além do diálogo familiar é consenso na literatura que também as atitudes e os comportamentos dos pais influenciam os filhos (Ferreira et al., 2013; Toni \& Silvares, 2016; Moscoso-Alvarez et al., 2016), o que obviamente inclui a sexualidade. Em El Salvador, as atitudes da família e dos amigos influenciaram o início da vida sexual dos adolescentes participantes de um estudo (Ruiz-Canela et al., 2012). Nele, a percepção de que os irmãos e amigos apoiam as relações sexuais esteve associada com maior probabilidade de os adolescentes praticarem sexo. Como fatores protetores do início da vida sexual estiveram: supervisão dos pais, receber mensagens de amigos ou irmãos apoiando a abstinência sexual e o apoio ao matrimônio por parte dos pais.

Adiciona-se ainda que o comportamento dos pais, tanto virtual quanto na vida fora da internet, induz à repetição do mesmo 
comportamento nos filhos. Lam e Wong (2015), por exemplo, mostraram que o vício de internet dos pais influenciou o vício de internet dos filhos. De forma semelhante, Raphaelli, Azevedo e Hallal (2011) mostraram que o uso de substâncias e uso de álcool pelos familiares aumentou a possibilidade de consumo pelos adolescentes. Por outro lado, a proximidade familiar, a relação positiva com os pais e o monitoramento parental funcionam como fatores de proteção para que os adolescentes não se envolvam em alguns comportamentos de risco, tais como abuso de álcool e atividade sexual sem preservativo (Kan, Cheng, Landale, \& McHale, 2010; Ciariano, Kliewer, \& Rabaglietti, 2009; Roche, Achmed, \& Blum, 2008).

É relevante ressaltar que a maioria destes estudos é de caráter quantitativo e não enfatizam as percepções dos adolescentes, além de se referirem aos contextos internacionais. No Brasil, em uma busca no portal da Coordenação de Aperfeiçoamento de Pessoal de Nível Superior - CAPES - com o descritor "sexting", foram encontrados dois artigos nacionais. Barros, Ribeiro e Quadrado (2014) analisaram reportagens de internet sobre o fenômeno e levantaram a questão de que, talvez, a fronteira entre o que é público e privado esteja fragilizada, o que levaria os adolescentes ao envolvimento em sexting. Por outro lado, Leal et al. (2017) relataram que os adolescentes participantes de seu estudo estavam familiarizados com o fenômeno, mas desconheciam o termo sexting. Além disso, chamaram a atenção para a culpabilização, especialmente das mulheres, quando do vazamento do conteúdo do sexting.

Diante disso, justifica-se a realização de pesquisas, especialmente qualitativas, para conhecer esse fenômeno em profundidade. Portanto, o objetivo desse estudo qualitativo e descritivo foi conhecer a percepção de adolescentes entre de uma escola pública da região metropolitana de Porto Alegre, sobre sexting e acerca do papel das relações familiares no fenômeno, pois a literatura supracitada aponta os reflexos da família nos comportamentos dos filhos, carecendo ainda saber se os filhos percebem tal associação em relação ao sexting.

\section{Método}

\section{Delineamento}

Foi realizada uma pesquisa qualitativa, de caráter exploratório e descritivo (Creswell, 2010). 


\section{Participantes}

Os indivíduos foram convidados a participar por meio de divulgação da pesquisa em uma escola pública da região metropolitana de Porto Alegre, com ou sem envolvimento em sexting. Seis adolescentes (Turato, 2008), três meninas e três meninos, com faixa etária entre 14 e 17 anos (Lei n. 8069, 1990) se inscreveram para participação no estudo.

\begin{tabular}{|c|c|c|c|c|c|}
\hline Participante & Idade & Série & Vive com: & $\begin{array}{l}\text { Já experienciou } \\
\text { sexting ativo? }\end{array}$ & $\begin{array}{c}\text { Já } \\
\text { experienciou } \\
\text { sexting } \\
\text { passivo? }\end{array}$ \\
\hline Entrevistada 01 & 16 & $\begin{array}{l}10 \text { Ano do } \\
\text { Ens. Médio }\end{array}$ & $\begin{array}{l}\text { Mãe, irmão } \\
\text { mais velho e } \\
\text { padrasto }\end{array}$ & Sim & Sim \\
\hline Entrevistada 02 & 15 & $\begin{array}{l}10 \text { Ano do } \\
\text { Ens. Médio }\end{array}$ & $\begin{array}{l}\text { Mãe e irmã } \\
\text { mais velha }\end{array}$ & Não & Sim \\
\hline Entrevistada 03 & 14 & $\begin{array}{l}9^{\circ} \text { Ano do } \\
\text { Ens. } \\
\text { Fundamental }\end{array}$ & $\begin{array}{l}\text { Pai, mãe e } \\
\text { irmão mais } \\
\text { velho }\end{array}$ & Não & Sim \\
\hline Entrevistado 04 & 17 & $\begin{array}{l}3^{\circ} \text { Ano do } \\
\text { Ens. Médio }\end{array}$ & $\begin{array}{c}\text { Mãe, irmã e } \\
\text { padrasto }\end{array}$ & Não & Sim \\
\hline Entrevistado 05 & 15 & $\begin{array}{l}10 \text { Ano do } \\
\text { Ens. Médio }\end{array}$ & Pai e mãe & Não & Sim \\
\hline Entrevistado 06 & 16 & $\begin{array}{l}2^{\circ} \text { Ano do } \\
\text { Ens. Médio }\end{array}$ & $\begin{array}{c}\text { Pai, mãe e } \\
\text { irmã mais } \\
\text { velha }\end{array}$ & Sim & Sim \\
\hline
\end{tabular}

\section{Aspectos éticos e instrumentos}

Após o fornecimento de autorização escrita por parte da direção da instituição e da aprovação do Comitê de Ética em Pesquisa da Universidade foi feita a divulgação da proposta na escola. Os participantes assinaram o Termo de Assentimento e trouxeram o Consentimento Livre e Esclarecido (TCLE) assinado pelo responsável legal.

O instrumento utilizado para a coleta de dados foi a entrevista semiestruturada, cujas perguntas foram elaboradas pelos autores: 1) Você conhece o termo sexting? O que você conhece sobre o compartilhamento de conteúdo sexual por adolescentes? 2) Quais seriam os motivos que levam os adolescentes a se envolverem em sexting? 3) Você conhece alguém que já vivenciou o envolvimento 
em sexting? Como foi essa experiência? Quais foram as consequências percebidas? Como reagiria se acontecesse com você? 4) $O$ que você pensa sobre as consequências para o adolescente? $E$ sobre as consequências para a família? 5) Como você percebe a relação entre o comportamento virtual dos pais, 0 tipo de monitoramento sobre a vida virtual dos filhos e o sexting?

\section{Procedimentos de coleta e análise dos dados}

Os seis adolescentes interessados se inscreveram na secretaria da escola, a qual passou os contatos para o pesquisador, que telefonou fornecendo as informações necessárias, explicando os objetivos e as etapas da pesquisa. Foi combinado horário em turno inverso a grade de aula de cada participante. As entrevistas foram gravadas em vídeo, pois melhora a compreensão do que foi dito pelos participantes, bem como auxilia na posterior transcrição das falas. Foram examinadas utilizando-se o método de análise de conteúdo (Bauer, 2008), agrupadas em categorias aposteriori (Gibbs, 2009) e à luz da teoria sistêmica (Nichols \& Schwartz, 2007).

\section{Resultados}

Após a análise de conteúdo foram elaboradas quatro Categorias Temáticas: Categoria I - Atitudes dos participantes sobre o sexting; Categoria II - Motivos que levam ao envolvimento em sexting ativo; Categoria III - Consequências para o adolescente e para a família do compartilhamento não autorizado do sexting; Categoria IV - Relações familiares e o envolvimento em sexting.

\section{Categoria I - Atitudes dos participantes sobre o sexting}

Os participantes não conheciam o fenômeno pelo termo sexting, mas relataram ser um comportamento comum entre os adolescentes. Todos disseram já ter se envolvido em sexting passivo, porém apenas E01 e E06 relataram terem enviado fotos suas a alguém que estavam namorando. Entretanto, E01 teve suas fotos divulgadas pelo então namorado sem sua permissão.

Com exceção da participante E03, os demais disseram não conhecer nenhum adolescente que nunca tenha, pelo menos, recebido sexting. Além disso, foi unânime a percepção de que o envolvimento em sexting passivo "não é um problema em si" (E05), mas sim o compartilhamento não autorizado do conteúdo deste, como exemplifica E06: "se tu namora alguém e tu confia muito [...] Não é uma coisa ruim". Entretanto, os entrevistados discordaram em relação à atitude diante da prática do sexting ativo. Para as três 
meninas entrevistadas, praticar sexting ativo, como disse E02: "é errado. [...] O que eu posso levar de bom disso?". Já para os demais entrevistados a prática tanto de sexting ativo quanto passivo foi vista como "normal" (E04, E05 e E06). Quanto à percepção dos participantes sobre quem mais produz e envia imagens de si mesmo, em relação ao sexo, não houve consenso.

\section{Categoria II - Motivos que levam ao envolvimento em sexting ativo}

Em relação aos motivos que levam o adolescente a enviar fotos nuas, foram descritas a impulsividade: "tipo tu não pensa na hora de mandar" (E01); o sentimento de que não iria acontecer nada de ruim: "Tu: - 'ah não devia, mas ah vou mandar. Não vai acontecer nada"' (E03); o desejo de atender ao pedido do namorado e a pressão dos pares: "mandam só pra agradar o namorado ou o amigo"(E04); busca por atividade sexual futura: "mando uma foto pra ela pra ver se ela se toca que eu quero transar com ela"(E05); expectativa de "receber imagens de volta"(E05); o sentimento de prazer em ver a foto do outro nu: "é só o prazer de ver a pessoa pelada"(E06); a busca por popularidade: "vai dar hã... mais curtida... vai 'bombar' mais"(E04); como forma "de brincadeira"(E04) entre amigos e para chamar atenção dos pais: "eu acho que é uma maneira de chamar atenção deles"(E03).

\section{Categoria III - Consequências para o adolescente e para a família do compartilhamento não autorizado do sexting}

Como consequências do compartilhamento do sexting para o adolescente, os participantes que não tiveram a experiência de terem suas imagens divulgadas, citaram o julgamento por parte dos amigos e familiares e a "fama ruim" (E02) que marca a história da pessoa: "serão lembradas de vadia, piranha, puta e coisas assim" (E03); o surgimento de mal-estar psicológico: "ficaria mal psicologicamente" (E01); tristeza e desespero: "ficar muito triste e desesperada" (E03). Além dessas percepções, a participante E01, que teve a experiência de ter suas imagens divulgadas, relatou que as consequências para ela foram o sentimento de vergonha e que se isolou socialmente: "eu nunca mais quis ficar perto das pessoas. Eu me afastei total. De todo mundo"; sentiu também o que relatou como "mal-estar psicológico [...] ]eu senti nojo de mim"; iniciou o uso de drogas e autolesão, "me envolvi com drogas e comecei a me cortar" e sofreu julgamento por parte de amigos e familiares "eles te julgam muito".

Os participantes relataram que as consequências para a família da pessoa que sofreu a exposição indevida seriam o surgimento de "raiva e vergonha [...] tristeza e decepção" (E02 e E03) nos pais. 
Também, que a família sofreria o julgamento dos outros: "o olhar pra essa família ficaria bem julgado" (E05).

\section{Categoria IV - Relações familiares e o envolvimento em sexting}

Em relação a como as relações familiares influenciam o envolvimento dos filhos em sexting não houve consenso entre os participantes. Os meninos percebem o envolvimento em sexting de forma diferente do que a família. Conforme E04: "Para os pais isso não é normal. Só que pros amigos eu acho que é bem mais normal, porque isso aí [o sexting] é muito famoso". Já as meninas disseram que tanto elas quanto seus pais veem o envolvimento no fenômeno como errado, como disse E02: "minha mãe ficaria muito decepcionada [...] ela acha muito errado".

Quanto à influência do comportamento dos pais na internet, alguns participantes disseram que "não tem nada a ver com o filho mandar" (E05). Já outros pensam que "se os pais mandassem nudez entre eles mesmos, daí eu acho que se o filho soubesse eu acho que influenciaria" (E04).

Quanto ao monitoramento da vida virtual dos filhos, os participantes disseram que, tanto o controle excessivo dos pais quanto o não monitoramento, ambos influenciam 0 filho em relação ao envolvimento em sexting ativo. Para eles, o controle excessivo da vida virtual dos filhos pelos pais pode incentivar o envolvimento do filho, pois os filhos "podem fazer de birra" (E05); "[...] aqueles pais que mais olham, tão sempre em cima, os filhos são os que mais fazem coisa errada" (E04). Por outro lado, o não monitoramento facilitaria tal envolvimento: "se deixar muito solto é mais fácil de eles mandarem" (E03). Portanto, o ideal, conforme os participantes E03 e E06 seria: "haver um equilíbrio".

Os participantes também relataram que a confiança mútua entre pais e filhos e o diálogo na família são fatores que influenciam a atitude dos filhos em relação ao sexting. Por exemplo, E02 disse ter muito diálogo na família entre ela, a irmã e mãe: "a gente conversa sobre tudo". A participante E03 disse que a presença afetiva da mãe e sua confiança são responsáveis por sua postura desfavorável sobre 0 sexting: "Minha mãe [...] ela sempre esteve comigo em todos os momentos e conversou comigo sobre todos os assuntos [...] é por isso que eu formei essa opinião". Já E01, que praticou sexting ativo e teve suas imagens divulgadas, relatou ausência de diálogo e distanciamento entre ela e os pais: "ninguém conversa com ninguém [...] tu [fala de sua mãe] não olha as minhas coisas e eu não olho as tuas". A falta de diálogo na família também descrita por E06: "a gente não conversa muito". 


\section{Discussão}

Os resultados mostram que, assim como descreveu Burkett (2015), apesar de o sexting ser praticado pelos adolescentes de forma crescente, eles não costumam utilizar este termo para se referir ao fenômeno. O mesmo aponta o estudo brasileiro de Leal (2017). Entretanto, é possível perceber que o envolvimento em sexting passivo se dá mesmo que o adolescente não queira, pois, como disseram os entrevistados, é comum receber sexting "mesmo sem pedir". Ademais, até mesmo os participantes cuja atitude diante do sexting ativo é de desaprovação receberam conteúdo sexual via smartphone. Esse resultado é corroborado pelo estudo de Strassberg et al., (2013), que descreveu que o sexting passivo foi mais comum do que o ativo. Esses achados indicam a relevância de pensar estratégias para informar adolescentes, familiares e escolas sobre a real possibilidade de que, mesmo aqueles adolescentes que não desejam, poderão receber conteúdo de sexting, assim como auxiliálos em como lidar com essa situação.

Os participantes não viram o envolvimento em sexting como ruim, não citando nenhuma consequência negativa do envolvimento no fenômeno, mas sim do compartilhamento não autorizado do conteúdo deste. Esse resultado é apoiado pela literatura (Assunção \& Matos, 2014; Mejía-Soto, 2014; Strassberg et al., 2013) e leva à conclusão de que o fenômeno em si não é um problema para os adolescentes, mas parece se encaixar dentro do desenvolvimento da vida sexual deles (Temple \& Choi, 2014) e servir como um meio para dar vazão às demandas que essa etapa do ciclo vital lhes impõe (Baumgartner et al., 2014; Macedo et al., 2010; Steinberg \& Morris, 2001). Todavia, o compartilhamento não autorizado leva à exposição pública e a consequências desagradáveis como isolamento, tristeza, vergonha e culpa, como relatou E01 e também o estudo de Leal (2017) com adolescentes do Sul do Brasil.

Cabe destacar que os entrevistados discordaram em relação à atitude diante da prática do sexting ativo. Para as três meninas entrevistadas, praticar sexting ativo "é errado", enquanto para os meninos a prática tanto de sexting ativo quanto passivo foi vista como "normal". Essa discordância pode ser pensada a partir do que descreveram Oliveira et al., (2014) sobre a expectativa dos pais para o início precoce da atividade sexual dos meninos a qual pode contribuir para que estes adquiram tal atitude em relação ao sexting ativo, já que os pais veem o início precoce da sexualidade dos meninos como símbolo de maturidade. Além disso, por meio da troca de conteúdo íntimo, o adolescente encontra uma maneira a mais de desenvolver sua sexualidade utilizando uma ferramenta tecnológica que faz parte do seu cotidiano (IBGE, 2018; Korenis \& Billick, 2014; Strassberg et al., 2013). 
Tal diferença de atitude entre os participantes se refletiu também no que eles disseram sobre os motivadores de envolvimento em sexting ativo. Para elas, um motivo de enviar imagens seria o desejo de fortalecer a intimidade dentro de um contexto de relacionamento amoroso, o que encontra apoio na literatura (Delevi \& Weisskirch, 2013; Drouin et. al., 2013; Henderson \& Morgan, 2011). Aliás, como se pode perceber, foi exatamente este o motivador do envio de imagens nuas da E01. Por outro lado, os meninos, semelhante ao que mostraram Delevi e Weisskirch (2013), descreveram como um motivador do sexting ativo o desejo de atrair alguém a uma possível atividade sexual futura. Já em relação aos motivadores que não envolvem relacionamento amoroso, a descrição dos participantes encontra eco em alguns estudos (Albury \& Crawford, 2012; Ringrose et al., 2012).

No que diz respeito às percepções dos participantes sobre as relações familiares e o envolvimento dos adolescentes em sexting, destaca-se o que disseram sobre a importância do diálogo na família e confiança mútua entre pais e filhos. Por exemplo, as entrevistadas 02 e 03 disseram existir diálogo na família e a presença de confiança mútua entre elas e seus pais, o que aponta para a existência de fronteiras nítidas (Nichols \& Schwartz, 2007). Elas relacionaram esses aspectos como importantes na definição de suas atitudes desfavoráveis em relação ao sexting ativo.

Por outro lado, os entrevistados E01 e E06, que se envolveram em sexting ativo, relataram haver falta de diálogo na família e a existência de fronteiras rígidas (Nichols \& Schwartz, 2007) entre eles e os pais, como disse E01: "ninguém conversa com ninguém". Apesar de não se poder aqui atribuir relação de causalidade, é necessário notar que a literatura apoia tais percepções, assinalando a importância da comunicação na família e o efeito protetor do diálogo familiar na vida dos adolescentes (Gomide et al., 2005; MoscosoAlvarez et al., 2016; Tomé et al., 2011). Apesar disso, as percepções dos entrevistados confirmam que os pais ainda têm dificuldade de dialogar sobre sexualidade com os filhos (González et al., 2017; Sevilla et al., 2016). Destaca-se a importância que as famílias sejam orientadas quanto à necessidade do estabelecimento de fronteiras nítidas com os filhos, uma vez que o distanciamento estabelecido pelas fronteiras rígidas na família foi percebido pelos adolescentes como um motivador de sexting ativo.

Embora a literatura mostre que os comportamentos dos pais, inclusive o que fazem na internet (Lam \& Wong, 2015) repercutem nos comportamentos dos filhos (Ferreira et al., 2013; Toni \& Silvares, 2016; Moscoso-Alvarez et al., 2016), não houve consenso entre os participantes a esse respeito. Alguns perceberam que aquilo que os pais fazem dentro e fora da internet não influencia o envolvimento dos filhos em sexting, enquanto outros disseram que sim. 
Adiciona-se ainda o que os participantes disseram quanto ao tipo de monitoramento dos pais sobre a vida virtual dos filhos e o sexting. Para os adolescentes, o envolvimento em sexting ativo pode ser influenciado pelo monitoramento excessivo de suas vidas virtuais pelos pais e também pelo não monitoramento, polos opostos do mesmo comportamento. Diante disso, é oportuno pensar que para o adolescente 0 não monitoramento dos pais pode significar distanciamento e desinteresse por parte deles em relação ao filho, fato que se observou no relato de E01 e seu consequente envolvimento em sexting ativo. Por sua vez, o monitoramento excessivo pode transmitir a sensação de sufocamento e controle, levando os filhos a "fazer de birra". Portanto, o ideal estaria no equilíbrio entre essas duas práticas, como referiram os próprios adolescentes.

Entretanto, especificamente quanto ao tipo de monitoramento dos pais e o envolvimento dos filhos em sexting ativo, não foram localizados estudos até o momento. Porém, a literatura indica que o monitoramento parental e a relação positiva com os pais funcionam como fatores de proteção para os adolescentes (Kanet al., 2010; Ciariano et al., 2009; Roche et al., 2008). Pode-se pensar, então, que, apesar de o envolvimento em sexting, tanto passivo quanto ativo, não ser percebido como problema para os participantes, um fator de proteção residiria na orientação dos pais quanto ao risco de exposição indevida que o envolvimento em sexting ativo traz consigo. Faz-se importante auxiliar o adolescente na percepção de que, uma vez enviado sexting, não se terá mais o controle sobre o que vai acontecer com seu conteúdo. Caso haja o compartilhamento não autorizado, isso pode trazer consequências para sua vida.

\section{Considerações Finais}

Esse estudo buscou investigar qualitativamente a percepção de adolescentes sobre sexting e suas associações com as relações familiares. Os resultados mostraram que o sexting entre adolescentes é um fenômeno comum. Também que, atualmente, é praticamente inevitável que, em algum momento, o adolescente se envolva no fenômeno. Por isso, destaca-se a importância da família em relação à atitude do adolescente frente ao sexting. Ela está diretamente implicada no fenômeno, especialmente se o adolescente sofrer exposição pública do conteúdo do sexting, pois os pais responderão legalmente por quaisquer implicações resultantes do compartilhamento não autorizado. Assim, sugere-se que os pais busquem meios de ampliar a comunicação na família e que a escola seja um espaço de fomento para tal, por meio de palestras, oficinas e discussões com adolescentes e familiares. 
Além disso, o ambiente familiar também deve ser visto como um importante lugar de fornecimento de informação e orientação sobre sexualidade para o adolescente. Assim, sugere-se que a atitude em relação ao sexting, tanto da família quanto dos educadores e dos terapeutas, seja de não qualificar o fenômeno em si como problema, pois ele faz parte da vivência da sexualidade dos adolescentes na atualidade. Antes, os profissionais e os pais devem orientar os adolescentes quanto aos riscos que o compartilhamento não autorizado do conteúdo do sexting e a consequente exposição pública trazem consigo. Sugere-se que, além de os pais conversarem com os filhos sobre sexualidade, sejam promovidos grupos de discussão entre os adolescentes na escola. Tais grupos podem propor atividades educativas para alertar outros adolescentes sobre a possibilidade de exposição da intimidade no mundo virtual e os orientar para o uso seguro das tecnologias de informação e comunicação.

Como limitações do estudo observa-se o número reduzido de seis participantes, não se podendo, assim, fazer afirmações generalizadas a partir dos dados aqui apresentados. Esse número talvez se explique pelo fato de que a participação demandaria uma exposição ao pesquisador, bem como à secretaria da escola, uma vez que era o local indicado de inscrição. Portanto, sugere-se a realização de pesquisas quantitativas que complementem os dados aqui expostos, verificando a prevalência do fenômeno entre os adolescentes de outras regiões brasileiras, identificando outras variáveis e suas associações com o sexting. Isso poderá auxiliar no melhor entendimento sobre o fenômeno e no desenvolvimento de estratégias para orientação de adolescentes, famílias, profissionais e educadores.

\section{Referências}

Albury, K., \& Crawford, K. (2012). Sexting, consent and young people's ethics: Beyond Megan's Story. Continuum: Jornal of media \& cultural studies, 26(3), 463-473. doi: $10.1080 / 10304312.2012 .665840$

Assunção, R. S., \& Matos, P. M. (2014). Perspectivas dos adolescentes sobre o uso do facebook: Um estudo qualitativo. Psicologia em estudo, 19(3), 539-547. doi:10.1590/141373722133716

Barros, S. da C., Ribeiro, P. R. C., \& Quadrado, R. P. (2014). Sexting: A espetacularização da sexualidade. Educação: Teoria e prática, 45(24), 197-215. doi:10.18675/1981-8106.vol24.n45.p197215

Bauer, M. W. (2008). Análise de conteúdo clássica: Uma revisão. In M. W. Bauer., \& G. Gaskell (Orgs.), Pesquisa qualitativa com 
texto, imagem e som: Um manual prático (pp. 189-217). Petrópolis, RJ: Vozes.

Baumgartner, S. E., Sumter, S. R., Peter, J., Valkenburg, P. M., \& Livingstone, S. (2014). Does country context matter? Investigating the predictors of teen sexting across Europe. Computers in human Behavior, 34, 157-164. doi:10.1016/j.chb.2014.01.041

Bezerra, M. A. R., Queiroz, M. V. O., \& Oliveira, K. N. S., (2014). Reflexões acerca do adolescer e da saúde no ambiente escolar. Journal of Human Growth and Development, 24(2), 175-180. Recuperado de http://pepsic.bvsalud.org/scielo.php?script=sci_arttext\&pid=S0 104-12822014000200009\&lng =pt\&tlng =pt

Brasil (1990). Lei no 8.069, de 13 de julho de 1990. Dispõe sobre o Estatuto da Criança e do Adolescente e dá outras providências. Brasília, DF: Diário Oficial da União. Recuperado de http://www.planalto.gov.br/ccivil_03/leis/L8069.htm

Burkett, M. (2015). Sex(t) Talk: A qualitative analysis of young adults' negotiations of the pleasures and perils of sexting. Sexuality \& Culture, 19(4), 835-863. doi:10.1007/s12119-0159295-0

Choi, E. P., Wong, J. Y., Lo, H. H., Wong, W., Chio, J. H., \& Fong, D. Y. (2016). The impacts of using smartphone dating applications on sexual risk behaviours in college students in Hong Kong. PLOS ONE, 11(11), 1-15. doi:10.1371/journal.pone.0165394

Ciariano, S., Kliewer, W., \& Rabaglietti, E. (2009). Adolescent risk behavior in Italy and the Netherlands: A cross-national study of psychosocial protective factors. European Psychologist, 14(3), 180-192. Recuperado

https://econtent. hogrefe.com/doi/abs/10.1027/10169040.14 .3 .180

Creswell, J. W. (2010). Projeto de pesquisa. Porto Alegre, RS: Artmed.

Delevi, R., \& Weisskirch, R. S., (2013). Personality factors as predictors of sexting. Computers in Human Behavior, 29, 25892594. doi: $10.1016 /$ j.chb.2013.06.003

Dir, A. L., Coskunpinar, A., Steiner, J. L., \& Cyders, M. A. (2013). Understanding differences in sexting behaviors across gender, relationship status, and sexual identity, and the role of expectancies in sexting. Cyberpsychology, Behavior and Social Networking, 16(8), 568-574. doi:10.1089/cyber.2012.0545

Döring, N. (2014). Consensual sexting among adolescents: Risk prevention through abstinence education or safer sexting?. Cyberpsychology: Journal of Psychosocial Research on Cyberspace, 8(1). doi:10.5817/CP2014-1-9 
Drouin, M., Vogel, K. N., Surbey, A., \& Stills, J. R. (2013). Let's talk about sexting, baby: Computer-mediated sexual behaviors among young adults. Computers in Human Behavior, 29, 2530. doi: $10.1016 / j$.chb.2012.12.030

Englander, E. (2012). Low risk associated with most teenage sexting: A study of 617 18-year-olds. Massachusetts Aggression Reduction Center, 1-12. Recuperado de http://www.marccenter.org

Ferreira, M., Nelas, P., Duarte, J., Grilo, C., Nave, F., \& Albuquerque, C. (2013). Family culture and adolescent sexuality. Aten Primaria, 45(1), 216-222. doi:10.1016/S0212-6567(13)700258

Gomide, P. I. C., Salvo, C. G. de, Pinheiro, D. P. N., Sabbag, G. M., Garcia, S. C., Brino, R. D. F., \& Thomazi, V. A. (2005). Correlação entre práticas educativas, depressão, estresse e habilidades sociais. Psicologia da Educação, 28, 23-50. doi:10.1590/S1413-82712005000200008

Gonçalves, R. C., Faleiro, J. H., \& Malafaia, G. (2013). Educação sexual no contexto familiar e escolar: Impasses e desafios. Holos, 29(5), 251-263. doi:10.15628/holos.2013.784

Gondim, P. S., Souto, N. F., Moreira, C. B., Cruz, M. E. C. da., Caetano, F. H. P., \& Montesuma, F. G. (2015). Acessibilidade dos adolescentes às fontes de informações sobre saúde sexual e reprodutiva. Journal of human growth and development, 25(1), 50-53. doi:10.7322/jhgd.96767

González, V., Orcasita, L. T., Carrillo, J. P., \& Palma-García, D. M. (2017).Comunicación familiar y toma de decisiones em sexualidad entre ascendientes y adolescentes. Revista Latinoamericana de ciencias sociales, niñez y juventud, 15(1), 419-430. doi:10.11600/1692715x.1512605022016

Gordon-Messer, D., Bauermeister, J. A., Grodzinski, A., \& Zimmerman, M. (2013). Sexting among young adults. Journal of Adolescent Health, 52, 301-306. doi: $10.1016 / j$.jadohealth.2012.05.013

Henderson, L., \& Morgan, E. (2011). Sexting and sexual relationships among teens and young adults. McNair Scholars Research Journal, 7(1), 31-39. Recuperado de https://scholarworks.boisestate.edu/mcnair journal/vol7/iss1/9

Instituto Brasileiro de Geografia e Estatística - IBGE. (2018, Dezembro). PNAD Contínua TIC 2017: Internet chega a três em cada quatro domicílios do país. Agência IBGE notícias. Recuperado de https://agenciadenoticias.ibge.gov.br/agenciasala-de-imprensa/2013-agencia-de-noticias/releases/23445pnad-continua-tic-2017-internet-chega-a-tres-em-cada-quatrodomicilios-do-pais 
Gibbs, G. (2009). Análise de dados qualitativos. Porto Alegre: Artmed.

Junqueira, R. D. (2018). A invenção da "ideologia de gênero": A emergência de um cenário político-discursivo e a elaboração de uma retórica reacionária antigênero. Psicologia política, 18(43), 449-502. Recuperado de http://pepsic.bvsalud.org/scielo.php?script=sci_arttext\&pid=S1 519-549X2018000300004\&lng =pt\&tIng = pt

Kan, M. L., Cheng, Y. A., Landale, N. S., \& McHale, S. M. (2010). Longitudinal predictors of change in number of sexual partners across adolescence and early adulthood. Journal of Adolescent Health, 46, 25-31. doi:10.1016/j.jadohealth.2009.05.002.

Korenis, P., \& Billick, S. B. (2014). Forensic implications: Adolescent sexting and cyberbullying. Psychiatric Quarterly, 85(1), 97-101. doi:10.1007/s11126-013-9277-z

Lam, L. T., \& Wong, E. M. Y. (2015). Stress moderates the relationship between problematic Internet use by parents and problematic Internet use by adolescents. The journal of Adolescent Health: Official publication of the society for adolescent medicine, 56(3), 300-6. doi: 10.1016/j.jadohealth.2014.10.263

Leal, L. das N., Rodrigues, G. S., Silveira, I. D. da., Amaro, T. V., Santos, D. B., \& Paludo, S. S. (2017). CEP em Selfie: Abordando sexting com adolescentes como forma de exposição virtual da sexualidade. Cadernos de Gênero e Diversidade, 1(3), 45-59. doi:10.9771/cgd.v3i1.17605

Livingstone, S., \& Görzig, A. (2014). When adolescents receive sexual messages on the internet: Explaining experiences of risk and harm. Computers in human behavior, 33, 8-15. doi: 10.1016/j.chb.2013.12.021

Macedo, M. M. K., Azevedo, B. H., \& Castan, J. U. (2010).Adolescência e Psicanálise. In M. K. Macedo (Org.). Adolescência e Psicanálise: intersecções possíveis (pp. 127148). Porto Alegre, RS: EDIPUCRS.

Mejía-Soto, G. (2014). Sexting: una modalidad cada vez más extendida de violencia sexual entre jóvenes. Perinatología y reproducción humana, 28(4), 217-221. Recuperado de http://www.scielo.org.mx/scielo.php?script=sci_arttext\&pid=S0 187-53372014000400007\&lng=es\&tIng=es

Morelli, M., Bianchi, D., Baiocco, R., Pezzuti, L., \& Chirumbolo, A. (2016). Sexting, psychological distress and dating violence among adolescents and young adults. Psicothema, 28(2), 13742. doi: $10.7334 /$ psicothema2015.193

Moscoso-Alvarez, M. R., Rodríguez-Figueroa, L., Reyes-Pulliza, J. C., \& Colon, H. M. (2016). Adolescentes de Puerto Rico: Una mirada a su salud mental y su asociación con el entorno familiar 
y escolar. Revista Puertorriqueña de Psicología, 27(2), 320-332. Recuperado de http://psycnet.apa.org/record/2016-49195-008 Nichols, M. P., \& Schwartz, R. C. (2007). Terapia Familiar: Conceitos e métodos. Porto Alegre, RS: Artmed.

Oliveira, N. de P., Béria, J. U., \& Schermann, L. B. (2014). Sexualidade na adolescência: Um estudo com escolares da cidade de Manaus/AM. Aletheia, (43-44), 129-146. Recuperado de http://pepsic.bvsalud.org/pdf/aletheia/n43-44/n4344a10.pdf

Ouytsel, J. Van, Ponnet, K., \& Walrave, M. (2017). Telematics and informatics adolescent sexting from a social learning perspective. Telematics and Informatics, 34(1), 287-298. doi:10.1016/j.tele.2016.05.009

Projeto de lei da 'Escola sem Partido' avança na Câmara e proíbe disciplinas sobre 'gênero' e 'orientação sexual'. (2018, Maio 9). G1. Recuperado https://g1.globo.com/educacao/noticia/projeto-de-lei-daescola-sem-partido-avanca-na-camara-e-proibe-disciplinassobre-genero-e-orientacao-sexual.ghtml

Raphaelli, C. O., Azevedo, M. R., \& Hallal, P. C., (2011). Associação entre comportamentos de risco à saúde de pais e adolescentes em escolares de zona rural de um município do Sul do Brasil. Cadernos de Saúde Pública, 27(12), 2429-2440. Recuperado de http://www.scielo.br/pdf/csp/v27n12/14.pdf

Rice, E., Rhoades, H., Winetrobe, H., Sanchez, M., Montoya, J., Plant, A., \& Kordic, T. (2012). Sexually explicit cell phone messaging associated with sexual risk among adolescents. Pediatrics, 130(4), 667-673. doi:10.1542/peds.2012-0021

Ringrose, J., Gill, R., Livingstone, S., \& Harvey, L. (2012). A qualitative study of children, young people and 'sexting': A report prepared for the NSPCC. National Society for the Prevention of Cruelty to Children, 5-71. Recuperado de https://www.researchgate.net/publication/265741962

Roche, K. M., Achmed, S., \& Blum, R. W. (2008). Enduring consequences of parenting for risk behaviors from adolescence into early adulthood. Social science \& medicine, 66, 2023-2034. doi:10.1111/j.1365-2214.2008.00864_4.x

Rood, C. J., Thackeray, J., Letson, M., Leder, R., \& Berlan, E. (2015). Prevalence of sexting, online solicitations, and offline meetings among adolescents of a large child advocacy center with suspected sexual abuse. Journal of Pediatric and Adolescent Gynecology, 28(2). doi:10.1016/j.jpag.2015.02.024

Ruiz-Canela, M., López-del Burgo, C., Carlos, S., Calatrava, M., Osorio, A., \& Irala, J. (2012). Familia, amigos y otras fuentes de información asociadas al inicio de las relaciones sexuales en adolescentes de El Salvador. Revista Panamericana de Salud 
Publica, 31(1), 54-61. Recuperado de https://scielosp.org/pdf/rpsp/2012.v31n1/54-61/es

Sevilla, T. M., Sanabria, J. P., Orcasita, L. T., \& Palma, D. M. (2016). Consistencies and discrepancies in communication between parents and teenage children about sexuality. Paidéia, 26(64), 139-147. doi: 10.1590/1982-43272664201601

Steinberg, L., \& Morris, A. S. (2001). Adolescent Development. Annual Review of Psychology, 52, 83-110. doi:00664308/01/0201-0083\$14.00

Strassberg, D. S., McKinnon, R. K., Sustaíta, M. A., \& Rullo, J. (2013). Sexting by high school students: An exploratory and descriptive study. Archives of Sexual Behavior, 42(1), 15-21. doi: 10.1007/s10508-012-9969-8

Temple, J. R., \& Choi, H. (2014). Longitudinal association between teen sexting and sexual behavior. Perspectives on Sexual and Reproductive Health, 47(5), 51-52. doi:10.1542/peds.20141974

Temple, J. R., Le, V. D., Berg, van den, P., Ling, Y., Paul, J. A., \& Temple, B. W. (2014). Brief report: Teen sexting and psychosocial health. Journal of Adolescence, 37(1), 33-36. doi:10.1016/j.adolescence.2013.10.008

Tolman, D. L., \& McClelland, S. I. (2011). Normative sexuality development in adolescence: A decade in review, 2000-2009. Journal of Research on Adolescence, 21(1), 242-255. doi:10.1111/j.1532-7795.2010.00726.x

Tomé, G., Camacho, I., Matos, G. M. de., \& Diniz, J. A. (2011). A influência da comunicação com a família e grupo de pares no bem-estar e nos comportamentos de risco nos adolescentes portugueses. Psicologia: Reflexão e Crítica, 249(4), 747-756. doi:10.1590/S0102-79722011000400015

Toni, C. G. D. S., \& Silvares, E. F. de M. (2013). Práticas educativas parentais e comportamentos de saúde e risco na adolescência: Um modelo preditivo. Psicologia Argumento, 31(400), 457. doi:10.7213/psicol.argum.31.074.AO01

Turato, E. R. (2008). Tratado da metodologia da pesquisa clínicoqualitativa: construção teórico-epistemológica, discussão comparada e aplicação nas áreas da saúde e humanas (5a ed.). Petrópolis, RJ: Vozes.

Vasconcellos, M. J. E. de (2013). Pensamento Sistêmico: o Novo Paradigma da Ciência.10 ed. Campinas, SP: Papirus.

Wolak, J., Finkelhor, D., \& Mitchell, K. J. (2012). How often are teens arrested for sexting? Data from a national sample of police cases. Pediatrics, 129(1), 4-12. doi:org/10.1542/peds.20112242 


\section{Endereço para correspondência \\ André Tavares Cardoso}

Universidade do Vale do Rio dos Sinos

Secretaria do Programa de Pós-Graduação em Psicologia

Av. Unisinos, 950, Cristo Rei, CEP 93.022-750, São Leopoldo - RS, Brasil

Endereço eletrônico: papoparalelo@gmail.com

\section{Denise Falcke}

Universidade do Vale do Rio dos Sinos

Secretaria do Programa de Pós-Graduação em Psicologia

Av. Unisinos, 950, Cristo Rei, CEP 93.022-750, São Leopoldo - RS, Brasil

Endereço eletrônico: dfalcke@unisinos.br

\section{Clarisse Pereira Mosmann}

Universidade do Vale do Rio dos Sinos

Secretaria do Programa de Pós-Graduação em Psicologia

Av. Unisinos, 950, Cristo Rei, CEP 93.022-750, São Leopoldo - RS, Brasil

Endereço eletrônico: clarissemosmann@gmail.com

Recebido em: $13 / 11 / 2018$

Reformulado em: 19/08/2019

Aceito em: $22 / 08 / 2019$

\section{Notas}

* Mestre em Psicologia, Terapeuta de Família e casal.

** Doutora em Psicologia. Professora titular do Programa de Pós-Graduação em Psicologia da Universidade do Vale do rio dos Sinos.

*** Doutora em Psicologia. Professora titular do Programa de Pós-Graduação em Psicologia da Universidade do Vale do rio dos Sinos.

Este artigo de revista Estudos e Pesquisas em Psicologia é licenciado sob uma Licença Creative Commons Atribuição-Não Comercial 3.0 Não Adaptada. 